\title{
Norois
}

Environnement, aménagement, société

\section{Évaluer la diversité des paysages de vallées. Réalisation d'une typologie en Basse-Normandie}

The measurement of a valley landscape diversity. Realization of a typology of valley landscapes on the Low-Normandy scale

\section{Marie-Anne Germaine}

\section{OpenEdition}

\section{Journals}

Édition électronique

URL : https://journals.openedition.org/norois/2253

DOI : $10.4000 /$ norois.2253

ISBN : 978-2-7535-1555-0

ISSN : $1760-8546$

Éditeur

Presses universitaires de Rennes

\section{Édition imprimée}

Date de publication : 1 juin 2008

Pagination : 7-19

ISBN : 978-2-7535-0717-3

ISSN : 0029-182X

\section{Référence électronique}

Marie-Anne Germaine, «Évaluer la diversité des paysages de vallées. Réalisation d'une typologie en Basse-Normandie », Norois [En ligne], 207 | 2008/2, mis en ligne le 01 juin 2010, consulté le 13 janvier 2022. URL : http://journals.openedition.org/norois/2253 ; DOI : https://doi.org/10.4000/norois.2253

(c) Tous droits réservés 


\title{
ÉVALUER LA DIVERSITÉ DES PAYSAGES DE VALLÉES \\ RÉAlisation D’une TyPologie en Basse-Normandie
}

\author{
Marie-Anne Germaine \\ GÉOPHEN - LETG UMR 6554 CNRS \\ (Université de Caen Basse-Normandie), \\ Esplanade de la Paix - 14032 CAEn cedex \\ marie-anne.germaine@unicaen.fr
}

\section{RÉSUMÉ}

En Normandie, comme dans l'ensemble du Nord-Ouest de la France, les vallées offrent des paysages singuliers et diversifiés qui rompent la monotonie des plateaux et collines environnants et qui donnent à la région une bonne part de ses traits physionomiques remarquables. À partir d'un diagnostic réalisé à l'échelle régionale pour caractériser les paysages de l'ensemble des vallées bas-normandes, nous proposons de mesurer leur diversité grâce à une démarche généralisable. Il s'agit d'une approche multiscalaire qui vise (1) à caractériser l'organisation interne du paysage à l'échelle du tronçon, unité élémentaire morphologiquement homogène composée d'un fond de vallée et de versants et isolée longitudinalement sur l'ensemble de la zone d'étude, et (2) à évaluer la singularité de ce paysage par rapport au milieu dans lequel il s'insère. Cette démarche s'appuie sur la combinaison de deux approches: une approche morphologique rendant compte de la structure du paysage (des " basses vallées » aux « gorges ») et une approche par traitement d'image renvoyant à la complexité des couvertures $d u$ sol. Des indicateurs quantitatifs résultent de ces deux étapes de traitement : ils traduisent les caractères visibles du paysage et permettent de caractériser de manière générique chacun des tronçons afin de mettre en place une typologie des paysages de vallées. La combinaison des deux approches permet de dégager les grands types de paysages qui marquent les vallées du nord ouest de la France. La prise en compte de cette hétérogénéité s'avère importante dans la définition de stratégies d'aménagement régional.

Mots CLÉs : Paysage - Vallée-Analyse spatiale-Morphologie - Télédétection-Occupation du sol-Indices paysagers.

\section{ABSTRACT}

The measurement of a valley landscape diversity. Realization of a typology of valley landscapes on the Low-Normandy scale

In Normandy, like in the northwest of France, valleys contain singular landscapes. Those are important in the structure of the regional landscape, and present an important landscape diversity. In order to characterize this landscape and measure their diversity, we propose a process which can be generalized to realize a regional diagnosis. This methodology aims (1) to measure the landscape internal diversity at "tronçon" scale - a homogeneous morphologic unit isolated longitudinally and consisting of a bottom valley and two slopes; and (2) to evaluate the singularity of this landscape in comparison to the environment 
in which it is inserted. This methodology is based on combination of two approaches: a morphologic one accounting for landscape structure (from "low valleys" to "gorges") and a remote sensing one revealing the land cover complexity. Quantitative indicators and landscape metrics are extracted at regional scale to build a valley landscape typology. The combination of these approaches enables to identify landscape types representative of heterogeneity of north-western valleys.

KEY WORDS : Landscape - Valley - Spatial Analysis - Morphology - Remote sensing - Land Use - Landscape metrics.

En Normandie comme dans l'ensemble du Nord-Ouest de la France, les vallées même petites constituent un trait fondamental du paysage. Ces espaces présentent une mosaïque de paysages variés, produits et révélateurs d'une histoire complexe et de mises en valeur singulières (Lespez et al., 2008). Abritant des milieux naturels remarquables et un patrimoine riche, ils offrent des paysages attractifs qui forment un cadre de vie de qualité assorti d'un fort potentiel récréatif. Espaces singuliers au fonctionnement distinct des plateaux et collines qui les entourent, les vallées font l'objet d'une patrimonialisation croissante. La multiplication des acteurs institutionnels, des mesures de protection et des opérations d'inventaires (RAMSAR, ZNIEFF, Natura 2000) qui s'y rapportent confirme l'élargissement de l'intérêt, d'abord porté aux grands fleuves (Val de Loire inscrit au patrimoine mondial de l'UNESCO, PNR des Boucles de la Seine), puis aux cours d'eau plus modestes. Les régions les plus urbanisées les reconnaissent comme « unités d'aménagement » parce qu'elles contribuent au maintien des grands équilibres écologiques et paysagers (IAURIF, 2000) mais plus généralement les gestionnaires tendent à en faire des entités paysagères spécifiques (Inventaire des paysages de Poitou-Charentes, 1999; Atlas des paysages de Wallonie, 2004). De plus, cet intérêt accru s'accompagne d'un changement d'attitude : les problématiques ne concernent plus seulement les sites d'exception mais portent aussi sur des enjeux globaux liés au maintien d'un cadre de vie de qualité. Bien que leur gestion se trouve confrontée d'une manière aigue aux problématiques inhérentes aux hydrosystèmes ainsi qu'à des enjeux multiples, les petites vallées rurales restent, elles, peu étudiées (Dupuis et Fischesser, 1997).

Dans ce contexte, il semble nécessaire de dresser l'état des paysages de vallées et d'évaluer la diversité de ces derniers. L'approche par la matérialité apparaît indispensable pour produire un diagnostic d'ensemble (Luginbühl, 2000) qui dépasse les approches thématiques à des échelles, de la vallée à la région, qui sont de plus en plus souvent celle des observatoires et des gestionnaires (Lespez et Ballouche, accepté). À travers ces travaux, il s'agit de proposer de nouvelles clés de lecture de ces paysages qui puissent être intégrées dans les démarches de gestion à différentes échelles et notamment à l'échelle régionale dans le cadre de la mise en œuvre des « trames bleues »/« trames vertes » ou de la directive européenne sur l'eau ${ }^{1}$.

Dans cet article est proposée une typologie paysagère construite à l'échelle d'un territoire régional représentatif des vallées du Nord Ouest de la France, de par sa position à l'interface entre Massif Armoricain et Bassin Parisien : la Basse-Normandie. Pour cela, la démarche mise en place s'appuie sur la caractérisation fine et systématique de ces espaces. Des indicateurs paysagers ont été extraits des données mobilisées (MNT et images SPOT) pour traduire de manière quantitative les caractères visibles du paysage. Ces derniers s'appuient sur la combinaison de deux étapes : une approche morphologique (Germaine et al., 2007) et la généralisation d'une approche des couvertures du sol d'abord menée sur une vallée représentative (Germaine et Puissant, accepté). Les résultats présentés montrent la typologie finale obtenue à l'échelle bas-normande avant de discuter de la diversité des vallées bas-normandes. 


\section{Méthodologie}

La démarche proposée pour mettre en place la typologie présente un caractère reproductible et s'appuie sur une approche multiscalaire (fig. 1 - planche I). La caractérisation générique des paysages de vallées repose sur la combinaison de critères morphologiques qui permettent d'appréhender la structure du paysage (Brierley et al., 2006; Fisher et al., 2004) et de critères liés aux couvertures du sol renseignées par traitement d'image (Hubert-Moy et al., 2003; Ducrot, 2005). Il s'agit de rendre compte de la matérialité du paysage à travers d'indicateurs quantitatifs et qualitatifs et ainsi passer d'une approche descriptive à une approche quantitative rarement utilisée pour évaluer le paysage en géographie (Luginbühl, 2000). Le paysage ne pouvant être décrit qualitativement par une approche de terrain pour l'ensemble des vallées à l'échelle de la région, il s'agit de «traduire de façon quantitative les termes utilisés pour caractériser le paysage » (Ducrot, 2005). La diversité de ces paysages est ainsi évaluée à travers plusieurs variables amenant à dégager de grands types en fonction de la topographie et de l'occupation du sol. Une typologie finale représentative des paysages des vallées du Nord Ouest de la France est alors proposée.

\section{EXTRACTION DE VARIABLES MORPHOMÉTRIQUES}

Les vallées sont extraites automatiquement à partir d'un modèle numérique de terrain (MNT) au pas de 50 mètres (CIGN) en tant que «forme en creux » (Germaine et al., 2007). Afin de rendre compte des contrastes rencontrés d'amont en aval et de mesurer la diversité inter- et intra-vallées, une sectorisation longitudinale est mise en place (Corbonnois et Zumstein, 1994; Béthemont, 1996). Il s'agit de dégager des entités paysagères cohérentes d'un point de vue morphologique : 95 tronçons sont identifiés manuellement le long de l'ensemble des vallées selon la variation de largeur du fond de vallée, les rapports de surface entre fond de vallée et versants et la distribution des pentes sur les versants (Germaine et al., 2007) (fig. la). L’objet « vallée » est donc appréhendé à travers le tronçon : entité fonctionnelle composée de trois unités - cours d'eau, fond de vallée et versants - et insérée dans un espace englobant (plateau/collines) pris en compte ici à travers une zone tampon d'un rayon de $5000 \mathrm{~m}$ - le milieu environnant - associée à chaque tronçon (fig. 1b).

La morphologie des vallées est caractérisée pour chaque tronçon par 13 variables morphométriques (fig. 1c) : des variables d'encaissement, des variables de forme et des variables longitudinales rendant compte du volume et de la forme des vallées ainsi que de la structure du réseau hydrographique (tableau 1). Une analyse en composantes principales (ACP) est réalisée sur l'ensemble de ces variables pour mettre en évidence la structure des tronçons. Puis une classification ascendante hiérarchique $(\mathrm{CAH})$ est effectuée dans le but de différencier de grands profils morphologiques de vallées (Germaine et al., 2007).

\section{Mise EN PLACE D’indiCES D'OCCUPATION DU SOL}

Les données génériques disponibles, telles Corine Land Cover (1/100 000), se révélant inadaptées à l'étude des paysages de vallées associées à de petits organismes fluviaux (ordre 2 à 4), une cartographie fine (1/15000) des modes d'occupation du sol est produite par traitement d'images satellites haute résolution $(5 \mathrm{~m})$ issues du capteur SPOT 5. Des classifications supervisées par maximum de vraisemblance ont été réalisées selon une typologie en 7 classes thématiques (eau, bâti, culture, prairie, bois dont haie, ripisylve et massif boisé) et confrontées à des échantillons de validation obtenus par photo-interprétation (Germaine, accepté; Germaine et Puissant, accepté). Les scènes SPOT mobilisées permettent de traiter de vastes espaces mais aussi de définir l'organisation interne des paysages de vallées et mettre en évidence des motifs ou combinaisons d'occupation du sol. Ainsi des informations sur la distribution et la répartition des modes d'occupation du sol ont pu être extraites à l'échelle des tronçons mais aussi des fonds de 
vallées et des versants. Elles ont ensuite été utilisées pour construire des indices choisis en référence aux notions employées dans les approches qualitatives basées sur des approches de terrain (Germaine et Puissant, accepté). Ces indices ont pour objectif de décrire les tronçons à travers leurs caractéristiques internes mais aussi celles de leur voisinage. Les vallées ne se définissent pas seulement par l'arrangement interne de leur paysage mesuré à l'échelle des fonds de vallées et des versants mais aussi par leur rapport à l'espace environnant. C'est pourquoi il est important de tenir compte du paysage dans lequel elles s'insèrent pour rendre compte de leur singularité à une échelle plus petite.

\begin{tabular}{|c|c|c|c|c|}
\hline \multicolumn{2}{|c|}{ Variables/Échelles } & Fond de vallée (FV) & Versants $(V)$ & Tronçon (T) \\
\hline \multirow{12}{*}{ 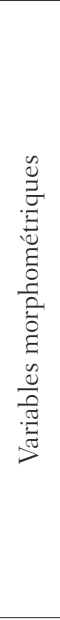 } & \multirow{5}{*}{ Pente } & & & Pente moyenne \\
\hline & & & & Écart-type de pente \\
\hline & & & Pente moyenne (rive droite) & \\
\hline & & & Pente moyenne (rive gauche) & \\
\hline & & & & Pente longitudinale \\
\hline & \multirow{4}{*}{ Forme } & & & $\begin{array}{l}\text { Largeur du rectangle } \\
\text { équivalent }\end{array}$ \\
\hline & & $\begin{array}{c}\text { Largeur du rectangle } \\
\text { équivalent } \\
\text { (FV principal) }\end{array}$ & & \\
\hline & & Part de & a surface & \\
\hline & & $\begin{array}{c}\text { Contribution des affluents } \\
\text { dans la surface du FV }\end{array}$ & & \\
\hline & \multirow{3}{*}{ Longitudinales } & & Ratio d'asymétrie des pentes & \\
\hline & & & & Densité de confluences \\
\hline & & & & Sinuosité du cours d'eau \\
\hline \multirow{11}{*}{ 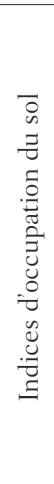 } & \multirow{5}{*}{$\begin{array}{l}\text { Organisation } \\
\text { interne }\end{array}$} & \multicolumn{2}{|c|}{ IF (fermeture) } & \\
\hline & & \multicolumn{2}{|c|}{ IU (emprise urbaine) } & \\
\hline & & \multicolumn{2}{|c|}{ ICP (ratio cultures/prairies) } & \\
\hline & & \multicolumn{2}{|c|}{ IdF (dispersion des bois) } & \\
\hline & & \multicolumn{2}{|c|}{ IdU (dispersion du bâti) } & \\
\hline & \multirow{4}{*}{ Singularité } & & & Indice de contraste IF \\
\hline & & & & Indice de contraste IU \\
\hline & & & & Indice de contraste ICP \\
\hline & & & & $\begin{array}{c}\text { Indice synthétique de } \\
\text { contraste }\end{array}$ \\
\hline & \multirow{2}{*}{ Spécifiques } & & & Accessibilité \\
\hline & & & & Caractère industriel \\
\hline
\end{tabular}

Tableau 1 : Liste des variables morphométriques et des indices d'occupation du sol List of morphometric variables and land cover metrics

Ainsi cinq indices sont construits pour caractériser l'organisation interne du paysage de chaque tronçon (tableau 1). Les trois premiers renvoient à la répartition des modes d'occupation du sol : la fermeture (IF) correspond à la combinaison du taux de boisement, du taux de ripisylve et de la densité de haies; l'emprise urbaine (IU) à la densité du bâti et le ratio cultures/prairies (ICP) à la part des prairies dans l'espace agricole. Les deux autres indices caractérisent la complexité de l'arrangement spatial de certains modes d'occupation du sol. Ils s'appuient sur des indices habituellement utilisés en écologie du paysage mesurés avec le logiciel CFRAGSTATS (McGarigal et Marks, 1995). Le logiciel propose le calcul de nombreux indices desquels il est souvent difficile de déduire l'information utile (Riitters et al., 1995; Cain et al., 1997), seul un indice de dispersion 
est retenu ici. Il permet de rendre compte de l'éclatement des taches qui composent le bois (IdF) et d'apprécier le caractère groupé ou dispersé de l'habitat (IdU) renseignant ainsi sur le caractère bocager ou non du paysage (Baudry et Burel, 1999). Les variables collectées sur les 95 tronçons présentent une distribution dissymétrique. Par exemple, la part occupée par les surfaces bâties est très variable selon qu'il s'agisse d'un tronçon urbain traversant une ville, d'un tronçon rurbain à proximité d'une agglomération ou d'un tronçon isolé en zone rurale. Une discrétisation en cinq classes est effectuée selon la méthode des seuils naturels : les bornes correspondent aux discontinuités identifiées sur chacune des séries et chaque classe se voit attribuer une valeur entre 1 et 5 . Ainsi, la fermeture est calculée par un indice (IF) qui correspond à la somme des valeurs attribuées aux variables, taux de boisement, taux de ripisylve, densité de haies, alors que les autres indices sont mesurés par la valeur d'une seule variable.

Le milieu dans lequel s'insère la vallée joue un rôle important puisqu'il participe à son identification et détermine le degré de singularité de son paysage : une vallée traversant un paysage de bocage n'a pas le même impact paysager qu'une vallée incisant le paysage ouvert d'un plateau céréalier. Cette dernière crée une rupture dans la monotonie du paysage par sa forme particulière de dépression mais également par la présence d'une couverture du sol originale. Des indices de contraste, confrontant les propriétés paysagères du tronçon de vallée à celles d'une zone tampon située autour, sont créés pour rendre compte de la singularité des paysages. Trois indices sont alors repris : la fermeture (IF), l'emprise urbaine (IU) et le ratio cultures/prairies (ICP) (Tableau 1). Chacun est mesuré à l'échelle du tronçon $(\mathrm{T})$ - fond de vallée et versants confondus - et à l'échelle de l'espace environnant (E). L'écart (T-E) entre les valeurs d'indices indique le degré de contraste entre les deux espaces. Ainsi l'indice de contraste fondé sur la comparaison du ratio cultures/prairies met, par exemple, en évidence :

- un contraste fort pour des tronçons de vallées herbagers $\left(\mathrm{ICP}_{\mathrm{T}}\right.$ élevé) insérés dans un paysage d'openfield ( $\mathrm{ICP}_{\mathrm{E}}$ faible) : exemple : « coulée verte » dans la Plaine de Caen;

-un contraste faible ou nul pour des tronçons de vallées herbagers ( $\mathrm{ICP}_{\mathrm{T}}$ élevé) qui traversent des milieux qui leur ressemblent $\left(\mathrm{ICP}_{\mathrm{E}}\right.$ élevé) : vallée herbagère dans le Bocage.

Un indice synthétique reprend la somme des valeurs absolues des écarts entre les trois indices : plus sa valeur est élevée, plus le tronçon traverse un paysage différent du sien traduisant un contraste paysager fort.

Afin de généraliser la démarche validée dans la vallée de la Seulles (Germaine et Puissant, accepté) et de l'étendre à l'échelle régionale, deux variables sont ajoutées pour rendre compte de caractères spécifiques de certaines vallées (tableau 1). D’une part, il semble important de tenir compte de l'accessibilité et de la visibilité des tronçons pour différencier les vallées facilement fréquentables le long desquelles des axes de communication permettent soit de longer le cours d'eau, soit d'accéder aux berges, soit d'avoir des points de vue sur la vallée, des vallées isolées dont les paysages apparaissent comme secrets ou cachés de par l'absence d'infrastructures de cheminement. Ce critère est mesuré par la présence de voies de communication longeant le cours d'eau. D'autre part, certaines vallées montrent un paysage très original lié à l'utilisation de la force hydraulique : il s'agit de vallées industrielles où le fond de vallée se présente comme une rue d'usines. Le caractère industriel des tronçons est pris en compte à travers un inventaire des sites industriels implantés en fond de vallée actifs ou encore visibles à l'état de friches.

Enfin, afin de révéler les motifs d'organisation des modes d'occupation du sol dans les vallées, l'ensemble des 16 indices collectés (tableau 1) est mobilisé dans une analyse factorielle des correspondances (AFC) suivie d'une $\mathrm{CAH}$ pour distinguer de grands types.

\section{Résultats : Des types de morphologies et de motifs d'occupation du sol à une typologie des paysages de vallées}

Chacune des deux étapes du diagnostic a permis de construire une typologie des tronçons de vallées que nous avons ensuite combinées. 


\section{UNE MAJORITÉ DE VALLÉES AUX FORMES BANALES}

Les résultats de l'ACP réalisée sur les variables morphométriques mettent en évidence le poids de l'encaissement dans la différenciation des tronçons. Ils sont traduits par 3 axes. L'axe 1, pour lequel les variables de pente participent à $73 \%$ oppose des tronçons à forte pente à des tronçons à pente faible. L'axe 2, qui repose à $58 \%$ sur des variables de forme, distingue les vallées larges des vallées étroites. Enfin l'axe 3, qui repose pour $65 \%$ sur la répartition des surfaces entre fond de vallée et versants, oppose des tronçons aux fonds de vallées étendus à des tronçons où les versants sont majoritaires. La CAH appliquée sur ces axes isole deux classes « extrêmes » rassemblant des tronçons aux formes remarquables, les « gorges » et les «basses vallées », et conduit surtout à regrouper la majorité des tronçons au sein d'une classe centrale (tableau 2) regroupant $85 \%$ des tronçons.

\begin{tabular}{|c|c|c|c|}
\hline Types & $\mathrm{a}-$ « Gorges » & b - «Vallées communes» & c - «Basses vallées» \\
\hline $\begin{array}{l}\text { Caractéristiques } \\
\text { (- forme, } \\
\text { - pente, } \\
\text { - répartition } \\
\text { versants/fond de } \\
\text { vallée, } \\
\text { - localisation) }\end{array}$ & $\begin{array}{l}\text { - Vallées encaissées } \\
\text { - Pentes très fortes } \\
\left(8,5 \text { à } 13^{\circ}\right) \\
\text { - Fonds de vallées } \\
\text { étroits ( } 3 \text { à } 16 \% \text { du } \\
\text { tronçon) } \\
\text { - Parties amont }\end{array}$ & $\begin{array}{c}\text { Caractéristiques morphologiques } \\
\text { «moyennes »(classe intermédiaire } \\
\text { majoritaire })\end{array}$ & $\begin{array}{l}\text { - Vallées larges à fond } \\
\text { plat } \\
\text { - Pentes très faibles } \\
\left(0,8 \text { à } 3^{\circ}\right) \\
\text { - Fonds de vallées } \\
\text { étalés }(42 \text { à } 72 \% \text { du } \\
\text { tronçon) } \\
\text { - Parties aval ou } \\
\text { secteurs de marais }\end{array}$ \\
\hline Effectif (tronçons) & 7 & $\begin{array}{c}80 \text { (plus de } 88 \% \text { de la superficie } \\
\text { des vallées) }\end{array}$ & 8 \\
\hline
\end{tabular}

Tableau 2 : Description des trois types de forme de vallées isolés par la CAH Description of the three valleys form types identified by ACH

Ces vallées qui présentent des caractéristiques morphologiques moyennes et fréquentes, sont les plus représentées parmi notre objet d'étude. Afin de mieux comprendre leur distribution en fonction de critères de forme et d'approfondir leur caractérisation, une seconde ACP, centrée uniquement sur ces tronçons aux formes banales, est réalisée à partir des mêmes variables. Le pourcentage d'information restitué par les quatre premiers axes est de $70 \%$. Les variables de pente, toujours les plus significatives, contribuent surtout à l'axe $1(79 \%)$ renvoyant aux variations d'encaissement des tronçons, alors que les autres variables ont un rôle plus important ici. L'axe 2 repose sur la répartition des surfaces entre fond de vallée et versants (63\%), l'axe 3 isole des tronçons présentant une forte incision (pente hydrographique forte) et une forte dissection des versants, enfin, l'axe 4 repose sur des variables longitudinales. La CAH appliquée sur ces quatre axes permet ensuite de dégager cinq classes au sein de la classe centrale. Ces classes homogènes se distinguent par leur pente et l'encaissement des vallées mais les variables de forme interviennent également dans la différenciation (tableau 3).

Des vallées très encaissées (tableau 3 : ba) se détachent avec des pentes qui demeurent fortes. Des vallées larges encaissées (tableau 3 : bb), regroupant seulement 3 tronçons, renvoient aux vallées très amples et rectilignes caractéristiques du Pays d'Auge. Des tronçons faiblement incisés et associés à des formes très évasées propres aux têtes de bassin versant sont identifiés comme des vallées peu marquées d'amont (tableau 3 : bd). La très faible incision des tronçons appartenant au dernier type (tableau 3 : be) amène à évoquer la présence de "vallées indistinctes ». Insérés dans une topographie englobante peu différenciée ou confondus dans le paysage environnant, ces tronçons ne présentent pas une forme de vallée bien identifiée. Enfin, le type médian des « vallées communes » (tableau 3 : bc) conserve un effectif conséquent. Ces tronçons s’inscrivent réellement dans le paysage environnant en tant que dépression mais ils ne présentent aucune caractéristique 
morphologique particulière et présentent alors des formes pouvant véritablement être qualifiées de «communes ». En fonction de l'encaissement des tronçons, 4 sous-types apparaissent néanmoins permettant de différencier ces vallées banales (tableau 3 : bc).

\begin{tabular}{|c|c|c|c|c|c|}
\hline Types & $\begin{array}{c}\text { ba - «Vallées très } \\
\text { encaissées » }\end{array}$ & $\begin{array}{c}\text { bb - «Vallées } \\
\text { larges } \\
\text { encaissées }\end{array}$ & $\begin{array}{l}\text { bc - «Vallées } \\
\text { communes » }\end{array}$ & $\begin{array}{c}\text { bd - «Vallées } \\
\text { peu marquées } \\
\text { d'amont » }\end{array}$ & $\begin{array}{l}\text { be - «Vallées } \\
\text { indistinctes » }\end{array}$ \\
\hline $\begin{array}{l}\text { Caractéristiques } \\
\text { (- forme, } \\
\text { - pente, } \\
\text { - répartition } \\
\text { versants/fond de } \\
\text { vallée, } \\
\text { - localisation) }\end{array}$ & $\begin{array}{l}\text { - Pentes fortes } \\
\left(4,7 \text { à } 6,5^{\circ}\right)\end{array}$ & $\begin{array}{l}\text { - Pentes fortes } \\
\text { - Forme large } \\
\text { - Forte dissection } \\
\text { des versants }\end{array}$ & $\begin{array}{l}\text { Caractéristiques } \\
\text { «moyennes } \\
\text { (encaissement } \\
\text { croissant entre les } \\
4 \text { sous-groupes) }\end{array}$ & $\begin{array}{l}\text { - Pentes faibles } \\
\left(2 \text { à } 3,9^{\circ}\right) \\
\text { - Ampleur } \\
\text { importante } \\
\text { - Amont }\end{array}$ & $\begin{array}{l}\text { - Pentes très } \\
\text { faibles }(1,5 \text { à } \\
\left.2,4^{\circ}\right) \\
\text { - Difficilement } \\
\text { identifiables } \\
\text { (faible forme en } \\
\text { creux) }\end{array}$ \\
\hline $\begin{array}{l}\text { Effectif } \\
\text { (tronçons) }\end{array}$ & 8 & 3 & 46 (4 classes) & 7 & 15 \\
\hline
\end{tabular}

Tableau 3 : Description des cinq types de forme de «vallées communes » isolés par la CAH Description of the five form types of " common valleys " identified by ACH

\section{UNE FORTE DIVERSITÉ DE MOTIFS DES COUVERTURES DU SOL DANS LES VALLÉES}

Les résultats de l'AFC menée sur les variables issues de l'approche des couvertures du sol mettent en évidence 4 axes. Ils concentrent $76 \%$ de l'information. L'axe 1, qui repose essentiellement sur des indices de fermeture $(27 \%)$ et de répartition des surfaces en culture ou en herbe (22\%) à l'intérieur des tronçons, oppose des tronçons à vocation herbagère très dominante à des tronçons boisés. L'axe 2, pour lequel l'indice de fermeture des versants contribue le plus (70\%), oppose des tronçons aux versants boisés à des tronçons aux versants ouverts. L'axe 3, plus complexe, repose principalement sur le caractère industriel (47\%) et l'indice d'emprise urbaine (25\%) mais aussi sur la dispersion de l'habitat (9\%) et le contraste d'emprise urbaine $(6 \%)$. Il différencie des tronçons industrialisés ou très urbanisés qui forment des couloirs d'urbanisation et de communication et des tronçons ruraux plus isolés où l'habitat est plutôt dispersé. Enfin, l'axe 4 repose sur les indices de dispersion des bois (39\%) et du bâti (18\%) et sur l'indice du ratio cultures/prairies (25\%) ainsi que sur l'indice de contraste global dans une moindre mesure. Il permet d'identifier des tronçons à habitat dispersé présentant des boisements diffus à des tronçons présentant les caractéristiques opposées et affichant un fort contraste avec leur milieu environnant. Il renvoie en fait à une distinction fonction de la localisation des tronçons dans le Bocage ou dans les campagnes ouvertes. La CAH menée sur ces axes fait ressortir huit grandes classes d'effectif variable (tableau 4).

Leur identification renvoie à trois facteurs principaux. Des densités de surface bâtie croissantes, la plus ou moins forte dispersion de l'habitat, une vocation de communication et un caractère industriel associés à des tronçons urbains (tableau $4: 7$ et 8) révèlent un gradient rural-urbain. La différenciation de tronçons boisés comportant de grandes étendues boisées (tableau $4: 1$ ), de tronçons bocagers à boisements diffus (Tableau $4: 2$ ), de tronçons herbagers (tableau $4: 4$ ) ou encore mixtes où l'arbre est peu présent met en évidence le degré de fermeture du tronçon. Enfin, la reconnaissance de tronçons boisés ou herbagers traversant des paysages ouverts formant de véritables « coulées vertes » (tableau $4: 6$ ) souligne l'importance de la singularité paysagère de certains tronçons par rapport à leur environnement. 


\begin{tabular}{|c|c|c|}
\hline Types & Caractéristiques & Effectif \\
\hline 1 - Vallées boisées & $\begin{array}{l}\text { - Bois massifs } \\
\text { - Bâti groupé } \\
\text { - Fort contraste IF }\end{array}$ & 15 \\
\hline 2 - Vallées boisées à herbagères & $\begin{array}{l}\text { - Bois assez massifs } \\
\text { - Bâti assez groupé } \\
\text { - Vocation herbagère plus forte qu'à l'extérieur } \\
\text { - Contraste IF et ICP assez importants } \\
\text { - Accès moyen }\end{array}$ & 23 \\
\hline 3 - Vallées bocagères rurales & $\begin{array}{l}\text { - Bois dispersé } \\
\text { - Bâti dispersé } \\
\text { - Vocation herbagère (tronçon et extérieur) } \\
\text { - Accès moyen }\end{array}$ & 21 \\
\hline 4 - Vallées herbagères & $\begin{array}{l}\text { - Fermeture faible } \\
\text { - Vocation herbagère marquée (plus qu'à l'extérieur) } \\
\text { - Rural }\end{array}$ & 10 \\
\hline 5 - Vallées bocagères urbanisées & $\begin{array}{l}\text { - Vocation herbagère forte (plus qu’à l'extérieur) } \\
\text { - Urbanisé (bâti groupé sur les versants) } \\
\text { - Bon accès }\end{array}$ & 3 \\
\hline 6 - «Coulées vertes » périurbaines & $\begin{array}{l}\text { - Très boisés, bois massifs } \\
\text { - Peu herbager mais plus qu'à l'extérieur (contraste ICP fort) } \\
\text { - Bâti dense } \\
\text { - Accès très bon }\end{array}$ & 8 \\
\hline 7 - Vallées urbaines & $\begin{array}{l}\text { - Peu boisés, bois massifs } \\
\text { - Contraste ICP fort } \\
\text { - Bâti très dense } \\
\text { - Accès très bon }\end{array}$ & 10 \\
\hline 8 - Vallées urbaines / industrielles & $\begin{array}{l}\text { - Très boisés, bois massifs } \\
\text { - Vocation herbagère } \\
\text { - Couloir d'urbanisation et de communication }\end{array}$ & 3 \\
\hline
\end{tabular}

Tableau 4 : Description des huit types de motifs d'occupation du sol dégagés par la CAH Description of the eight landscape types identified by $\mathrm{ACH}$

\section{TyPologie FinALE ET CARTOGRAPHIE DES TYPES DE PAYSAGES DE VALLÉES}

La figure 2 présente la combinaison des résultats de l'approche morphologique dont les 7 types sont reportés en abscisses, et de l'approche des occupations du sol dont les 8 types sont reportés en ordonnées. Des 56 associations possibles de ce croisement, la moitié n'existe pas. Des simplifications sont apportées pour réduire le nombre de sous-types de 28 à 11 en supprimant les classes à un seul individu et en regroupant les sous-types proches (fig. 2).

Des combinaisons privilégiées apparaissent. En particulier, les types morphologiques remarquables, gorges et basses vallées, affichent une faible dispersion. Les vallées industrielles sont toujours localisées dans les gorges (fig. 2: A). Les vallées les plus encaissées sont boisées (fig. 2 : B). À l'inverse, les basses vallées ont une vocation herbagère et renferment le plus souvent des paysages de marais ou d'estuaires (fig. 2: C) sauf sur les littoraux les plus touristiques où les basses vallées sont urbanisées (fig. 2 : D).

Si les types évoqués précédemment s’individualisent clairement, une dispersion plus forte se remarque autour des types morphologiques intermédiaires. Néanmoins, les vallées larges encaissées se démarquent avec des paysages boisées à bocagers (fig. $2: G$ ), ainsi que deux types de vallées aux formes « indistinctes » qui se distinguent ensuite selon qu'elles soient boisées à bocagères plutôt rurales (fig. 2 : E) ou urbanisées (fig. 2 : F).

En revanche, les vallées les plus nombreuses (52 tronçons) qui possèdent les formes les plus communes (tableau 3 : bc) sont aussi celles qui présentent les motifs d'occupation du sol les 


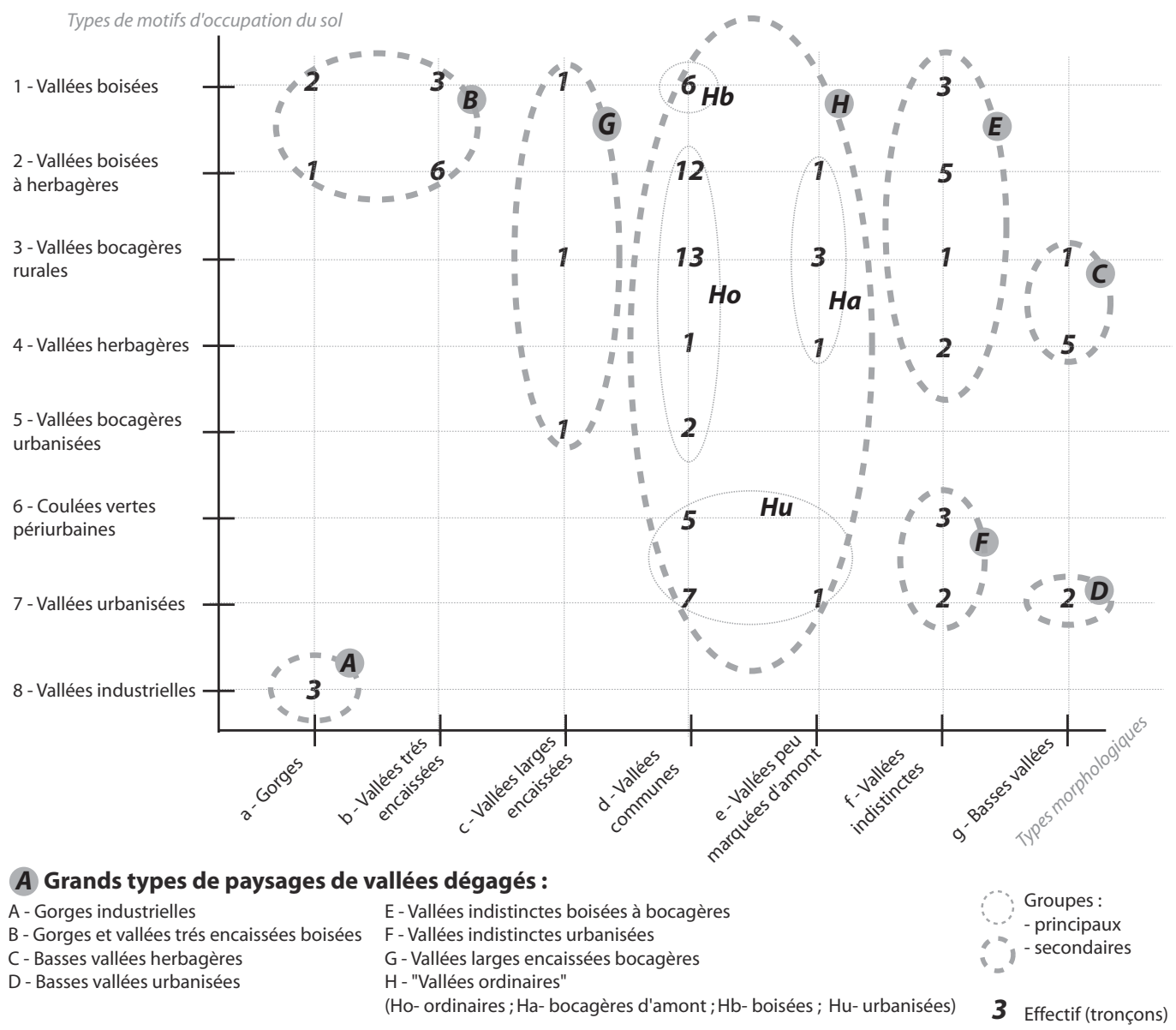

Figure 2 : Combinaison des deux typologies : répartition des tronçons et distinction de grands types paysagers de vallées Combination of two typologies: "tronçons" distribution and landscape types's differentiation

moins singuliers (tableau $4: 2$ et 3). Il s'agit de vallées plutôt rurales, assez boisées et à vocation herbagère. Ces tronçons peuvent être qualifiés de « paysages ordinaires » (Dewarrat et al., 2003) : leur forme est banale et l'organisation de leur couverture du sol renvoie à des paysages récurrents dans tout le Nord Ouest de la France. Néanmoins la confrontation des deux typologies permet de dégager des spécificités au sein de cette classe centrale. Autour d'un noyau central de «vallées ordinaires » composé des 28 tronçons (fig. 2, Ho) gravitent des groupes périphériques aux caractéristiques paysagères plus singulières. On distingue ainsi des tronçons boisés (fig. 2, Hb), des tronçons apparaissant comme de véritables « coulées vertes » périurbaines à urbanisées (Hu) ou encore des tronçons bocagers aux morphologies moins marquées car localisés en section amont (fig. 2 : Ha). Finalement, il est possible de dégager 8 grands types de paysages de vallées (fig. 3 - planche II).

\section{Discussion}

La démarche proposée s’appuie sur des méthodes simples (télédétection, analyse multivariée) et généralisables pour caractériser les paysages de vallées de l'échelle du tronçon à l'ensemble 
d'un territoire régional. Elle souligne la complémentarité, souvent adoptée par les géographes des approches morphologique et couverture du sol dans le cadre d'analyses paysagères (Wieber, 1980 ; Deffontaines, 1986 ; Bontron et al., 1991 ; Joliveau, 2004) mais aussi l'intérêt de l'approche quantitative pour rendre compte de l'état du paysage. La constitution de cette base d'information homogène couvrant l'ensemble de la région a permis de proposer la mise en place d'indices : ceuxci expriment de manière générique les propriétés du paysage visible permettant ainsi de passer d'une approche descriptive à une approche quantitative. Inspirés des approches géographiques classiques appuyées sur des analyses en mode raster mais aussi des concepts de l'écologie du paysage, ils permettent de caractériser les paysages de vallées de l'échelle locale à régionale. Ils autorisent la conduite d'études comparatives, soit pour construire des typologies de paysage comme ici, soit pour évaluer des dynamiques paysagères dans le cadre d'analyses diachroniques. Enfin, l'ensemble des étapes s'appuie sur des méthodes reproductibles et peut s'appliquer à l'étude du paysage d'autres objets géographiques formant des continuums écologiques ou des corridors aux aménités spécifiques et aux forts enjeux écologiques et récréatifs, permettant alors de mieux prendre en compte leur singularité dans les démarches de planification.

Le diagnostic mené en Basse-Normandie met d'abord en avant l'importance des espaces de vallées dans le paysage régional (42\%). La typologie finale affirme ensuite la diversité de ces paysages à l'échelle régionale (fig. 3). L'approche morphologique comme «biophysique » démontre une grande variété de formes. Deux types emblématiques mettent en évidence l'importance du facteur morphologique : les basses vallées herbagères et les gorges et vallées très encaissées boisées (auxquelles peuvent être associées les gorges industrielles), qui renvoient aux rares paysages de vallées reconnus dans l'Inventaire des paysages de Basse-Normandie réalisé pour la DIREN (Brunet, 2001). Parmi les 75 unités de paysage qui y sont répertoriées, seules 9 renvoient explicitement à des paysages de vallées dont 3 dans la famille «les paysages de marais » et 3 dans le sous-type «Les vallées en gorges ». Le fait qu’il s'agisse presque toujours de vallées appartenant aux deux types remarquables isolés lors du diagnostic montre l'importance de la morphologie dans la représentation des paysages de vallées (Germaine et al., 2007). La diversité de paysages mise en avant par la typologie renvoie en partie aux grands traits de l'organisation géographique régionale. Ainsi, les basses vallées herbagères des sections aval occupent le littoral et les grandes zones de dépression des marais de Carentan ou de la Dives tandis que l'arc des hauteurs du synclinal bocain apparaît clairement renvoyant aux gorges et vallées très encaissées boisées qui le traversent - Suisse Normande, Gorges de la Vire... - (fig. 3).

La typologie met surtout en évidence la diversité de paysages de vallées souvent négligées par les approches classiques et jamais directement évoquées en tant que telles. Ces vallées, qui renvoient aux cinq types les plus banals de la typologie, ne sont pas reconnues comme paysage à part entière dans les approches classiques mais le plus souvent intégrées dans des entités paysagères englobantes qui ont pour effet de négliger leur spécificité.

Les « vallées larges encaissées bocagères », qui renvoient au Pays d’Auge et à la vallée de la Sée (fig. 3), se distinguent parmi celles-ci : bien qu'elles ne soient jamais mises en avant, elles sont bien perçues comme structurantes. Dans le Bocage, seules ces vallées sont présentent dans les descriptions classiques dans la mesure où elles révèlent le paysage à l'observateur. C'est le cas pour « les grandes vallées augeronnes » par exemple qui par leur amplitude et leurs longs versants participent largement à la structuration du paysage bocager « en tableaux » (Brunet, 2001, p 411) ou bien « le val de Sée dans son écrin » (2001, p 541) qui structure également le paysage.

La typologie met ensuite en évidence des vallées urbanisées, ordinaires ou indistinctes (fig. 3). Leur répartition montre une forte concentration dans la Plaine de Caen. La structuration de l'espace régional joue sans aucun doute un rôle important dans la localisation de ces tronçons concentrés à proximité de Caen et des littoraux touristiques (Côte de Nacre). Par ailleurs, l'originalité de ces vallées est manifeste par la rupture qu'elles apportent par leur forme en creux dans le paysage monotone des plateaux (Seulles, Orne inférieure, Dan, Guigne...). L'usage du sol est également spécifique, les prairies permanentes s'y replient dans un espace environnant largement ouvert. 
La prise en compte de cette spécificité paysagère est sans doute importante pour comprendre les processus d'étalement urbain depuis l'agglomération caennaise car il semble se diffuser de façon privilégiée dans ces petites vallées banales devenues des lieux de vie convoités.

Enfin, la typologie met en avant la complexité des paysages des «vallées ordinaires » rurales. Sur la figure 3, apparaissent ces vallées bocagères qui transgressent les ensembles géographiques en s'insinuant aussi bien dans la partie sédimentaire qu'armoricaine de la région. Ces tronçons présentent une diversité : ils renvoient à des vallées marquées bien qu'ordinaires, aux parties amont localisées dans les zones schisteuses des collines armoricaines ou à des vallées indistinctes peu visibles dans le paysage environnant. Ils sont le plus souvent marginalisés dans la mesure où ils n’imposent pas leur forme ou leur singularité dans le milieu dans lequel ils s'insèrent. Pourtant des enjeux spécifiques amènent à s'interroger sur les usages et modes de gestion actuels de ces versants et fonds de vallées face aux menaces de déprise agricole et de biodiversité.

\section{Conclusion et perspectives}

À travers une démarche reproductible et la mise en place d'indicateurs génériques traduisant les caractères visibles du paysage, le diagnostic proposé offre une meilleure identification de l'objet «vallée » et rend compte de sa diversité. Il met en particulier en évidence une complexité souvent non reconnue : celle des vallées aux formes banales, les plus nombreuses et les plus souvent ignorées. Révélant de la sorte l'intérêt de ces espaces et posant la question de leurs identités et de leurs fonctions, la typologie permet d'identifier et de localiser des espaces variés aux vocations très différentes : agricole, résidentielle, industrielle ou récréative. Sont également repérées des zones de concentration d'enjeux (paysages fermés sous forte emprise urbaine ou paysages agricoles en déprise), des paysages marqués par des dynamiques fortes par le passé (vallées industrielles) ou actuellement («coulées vertes » périurbaines). Ce sont ainsi toutes les nuances et la complexité des «vallées ordinaires » récurrentes dans tout le Nord Ouest de la France qui sont mises en évidence et qui peuvent alors être intégrées dans les démarches de gestion à différentes échelles. La typologie finale invite alors à s'interroger sur l'histoire et les mécanismes de fonctionnement propres à chacun des grands types de paysages de vallées. Afin de mieux cerner cette diversité et de comprendre les systèmes paysagers contemporains associés, des tronçons représentatifs de la diversité régionale vont faire l'objet d'un diagnostic à l'échelle locale. C'est à partir de ces zones tests et d'une enquête de terrain auprès des acteurs que pourra alors s'engager une réflexion sur l'identité de ces paysages notamment par la confrontation de l'objet et de ses représentations. Il sera alors possible d'identifier les enjeux auxquels ces paysages sont soumis de l'échelle locale à régionale et de mesurer sur le terrain cette diversité révélatrice de problématiques fortement différenciées.

\section{Bibliographie}

Baudry (J.), Burel (F.), 1999. - Écologie du paysage, Concepts, méthodes et applications, Paris, TEC \& DOC, $362 \mathrm{p}$.

Bethemont (J.), Andriamahefa (H.), Rogers (C.), Wasson (J. G.), 1996. - « Une approche régionale de la typologie morphologique des cours d'eau », Revue de géographie de Lyon, Géocarrefour, vol. 71, n 4 , p. 311-322.

Bontron (J.-C.), Luginbühl (Y.), Cros (Z.), 1991 - Méthodologie pour l'identification et la typologie des paysages, Paris-la-Défense, DAU, 129 p.

Bornand (M.), Robbez-Masson (J. M.), Donnet (A.), Lacaze (B.), 1997. - « Caractérisation des sols et paysages des garrigues méditerranéennes. Typologie et extrapolation spatiales par traitement d'images satellitaires », Étude et Gestion des Sols, 4 (1), p 27-42. 
Brierley (G.), Fryirs (K.), Vikrant (J.), 2006. - « Landscape connectivity : the basis of geomorphic applications ", Area, vol.38-2, p. 165-174.

Brunet (P.), avec la collaboration de Girardin (P.), 2001. - Inventaire des paysages de Basse-Normandie, Conseil Régional de Basse-Normandie, DIREN Basse-Normandie, Caen, 2 Tomes, 871 p.

Cain (D. H.), RitTters (K.), Orvis (K.), 1997. - «A multi-scale analysis of landscape statistics », Landscape Ecology, vol. 12, p 199-212.

Conseil de L'Europe, 2000. - Convention européenne du paysage, Florence, 18 p.

Conservatoire d'espaces naturels de Poitou-Charentes, 1999. - Inventaire des paysages de Poitou-Charentes, [http://www.paysage-poitou-charentes.org/].

Corbonnois (J.), Zumstein (J.-F.), 1994. - « Proposition de typologie des cours d'eau. Application au réseau hydrographique du Nord-Est de la France (bassin de la Moselle) », Revue de géographie alpine, n² p. 15-24.

DefFontaines (J.-P.), 1986. - « Un point de vue d'agronome sur le paysage. Une méthode d'analyse du paysage pour l'étude de l'activité agricole », dans Lectures du paysage, Paris, Foucher/INRAP-Dijon, p. 35-52.

Dewarkat (J.-P.), Quincerot (R.), Weil (M.), WoefFray (B.), 2003. - Paysages ordinaires, de la protection au projet, Mardaga, Liège, 95 p.

Ducrot (D.), 2005. - Méthodes d'analyse et d'interprétation d'images de télédétection multi-sources, Extraction de caractéristiques du paysage, Mémoire d'Habilitation à Diriger des Recherches, INP Toulouse, 240 p.

Dupuis (M.-F.), Fischesser (B.), 1997. - « Richesse, diversité et enjeux des paysages de rivière », Ingénieries EAT, numéro spécial Rivières et paysages, p 11-31.

Feltz C. (dir.), Droeven, E., Kummert M., 2004. - «Les territoires paysagers de Wallonie », Études et Documents CPDT 4, Jambes, Ministère de la Région wallonne, DGATLP, 68 p + Cartes.

Fischer (P.), Wood (J.), Cheng (T.), 2004. - «Where is Helvellyn? Fuzziness of multi-scale landscape morphometry », Royal Geographical Society, vol. 29, p 106-128.

Germaine (M.-A.), (à paraître). - « Vers une gestion intégrée des "paysages ordinaires" des petites vallées du nord-ouest de la France : l'exemple de la vallée de la Seulles », Bulletin de l'Association des Géographes Français, 11 p.

Germaine (M.-A.), Puissant (A.), (à paraître). - « Extraction d'indices paysagers et analyse quantitative des paysages de "vallées ordinaires" à partir de données images : L'exemple de la Seulles (Calvados, France) », Cybergéo, 15 p.

Germaine (M.-A.), Puissant (A.), Lespez (L.), Ballouche (A.), 2007. - «Analyse spatiale et typologie morphologique des petites vallées bas-normandes », Revue Internationale de Géomatique, vol. 17, 3/4, p 415-430.

Hubert-Moy (L.), Clément (B.), Lennon (M.), Houet (T.), Lefauvre (E.), 2003. - « Étude de zones humides de fond de vallées à partir d'images hyperspectrales CASI : Application à un bassin versant de la région de Pleine-Fougères (Bretagne, France)», Photo-Interprétation, vol. 39, p. 33-43.

IAURIF, 2000. - « Les vallées d'Île-de-France : Renouer avec le fil de l'eau », Cahiers de l'IAURIF, n 125 , $239 \mathrm{p}$.

Jenness (J.), 2005. - Documentation «Topographic Position Index », [http://jennessent.com], 41 p.

JoLiveau (T.), 2004. - Géomatique et gestion environnementale du territoire. Recherches sur un usage géographique des SIG, Mémoire d'Habilitation à diriger des recherches, Rouen, Université de Rouen, 2 vol., $504 \mathrm{p}$.

Joliveau (T.), Michelin (Y.), 1998. - «Approche méthodologique de la gestion paysagère concertée d'un espace avec un système d'information géographique : l'exemple de la commune de Viscomtat » (63), dans Actes du Colloque Gestion des territoires ruraux : connaissances et méthodes pour la gestion publique, 27 et 28 avril 1998, Clermont-Ferrand/Cachan, Cemagref, p 85-102.

Lespez (L.), Cador (J.-M.), Carpentier (V.), Clet-Pellerin (M.), Germaine (M.-A.), Garnier (E.), MarCiGNY (C.), 2008. - « Trajectoire des paysages des vallées normandes et gestion de l'eau, du Néolithique aux enjeux de la gestion contemporaine », dans GALOP (D.) (dir.), Paysage et environnement : de la reconstitution du passé aux modèles prospectifs, Besançon, Presses Universitaires de Franche-Comté, coll. «Annales littéraires », série « Environnement, Société et Archéologie», p 61-75. 
LuginbüHL (Y.), 2000. - « Le paysage, le qualitatif et le quantitatif », Politiques publiques et paysages, Actes du séminaire d'Albi, 28-30 mars 2000, p. 59-64.

MCGARIGAL (K.), MARKS (B. J.), 1995. - FRAGSTATS : spatial pattern analysis program for quantifying landscape structure, USDA For. Serv. Gen. Tech. Rep. PNW-351.

Montembault (D.), 2002. - Les vallées face à l'appropriation urbaine, Des mutations de l'occupation du sol dans les grandes vallées proches d'Angers aux nouveaux paysages, Thèse de doctorat de géographie, Angers, 2 Tomes, $409 \mathrm{p}$.

Rittters (K. H.), O’neill (R.V.), Hunsaker (C. T.), Wichham (J. D.), Yankee (D. H.), Timmins (S. P.), JONES (K. B.), JACKSON (B. L.), 1995. - «A factor analysis of landscape pattern and structure metrics », Landscape Ecology, vol. 10, p. 23-39.

Robbez-Masson (J.-R.), 1994. - Reconnaissance et délimitation de motifs d'organisation spatiale : application à la cartographie des pédopaysages, Thèse de doctorat ENS. M, Montpellier, 161 p.

WeISS (A.), 2001. - Topographic Position and landforms analysis, ESRI International User Conference, San Diego, CA.

Wieber (J.-C.), 1980 - «Établissement d'un modèle régional de classification typologique des paysages », $B A G F, \mathrm{n}^{\circ}$ 468, p 127-129.

\section{Remerciements}

L'auteur tient à remercier le Conseil Régional de Basse-Normandie qui soutient ce programme de recherche ("La dynamique des paysages de vallées - Enjeux et perspectives. Exemples bas-normands »; Novembre 2005 à Novembre 2008; resp. A. Ballouche et L. Lespez); le CNES par qui ont pu être acquises des scènes SPOT 5 dans le cadre du programme ISIS; Anne Puissant pour les conseils en télédétection et statistiques; ainsi que les relecteurs et le comité de rédaction pour nous permettre de publier les résultats de ces travaux.

Cet article a été reçu le 6 décembre 2007 et définitivement accepté le 15 mai 2008. 
Planche I (Marie-Anne GERMAINE - Évaluer la diversité des paysages de vallées...)

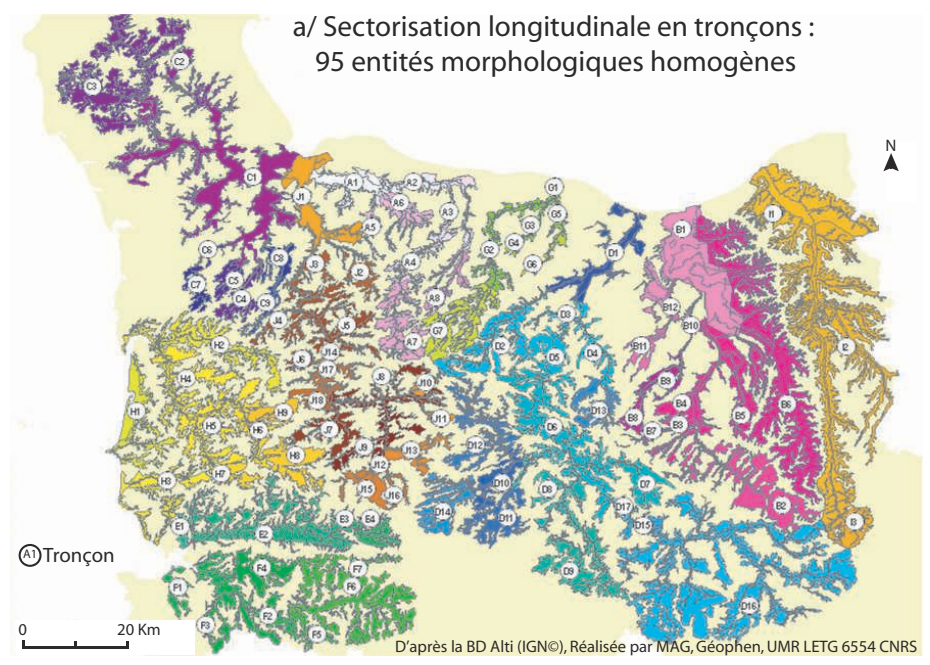

b/ Une démarche multiscalaire
adaptée à l'objet "vallée"
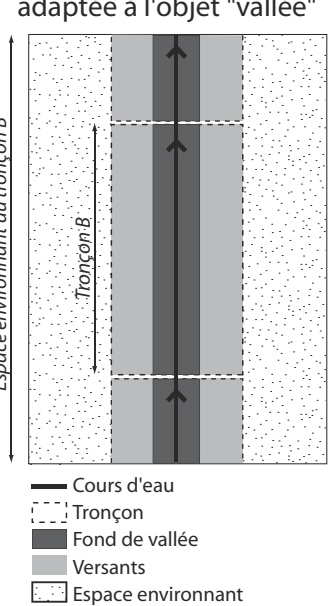

c/ Méthode de diagnostic

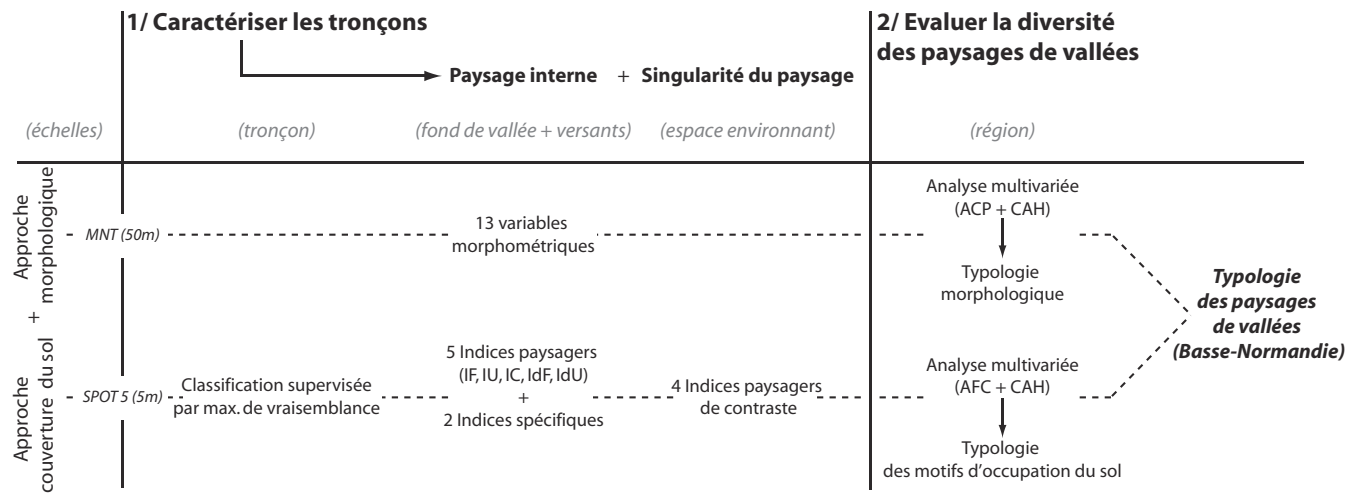

Figure 1 : Terrain d'étude et démarche de recherche Study area and methodology 
Planche II (Marie-Anne GERMAINE - Évaluer la diversité des paysages de vallées...)

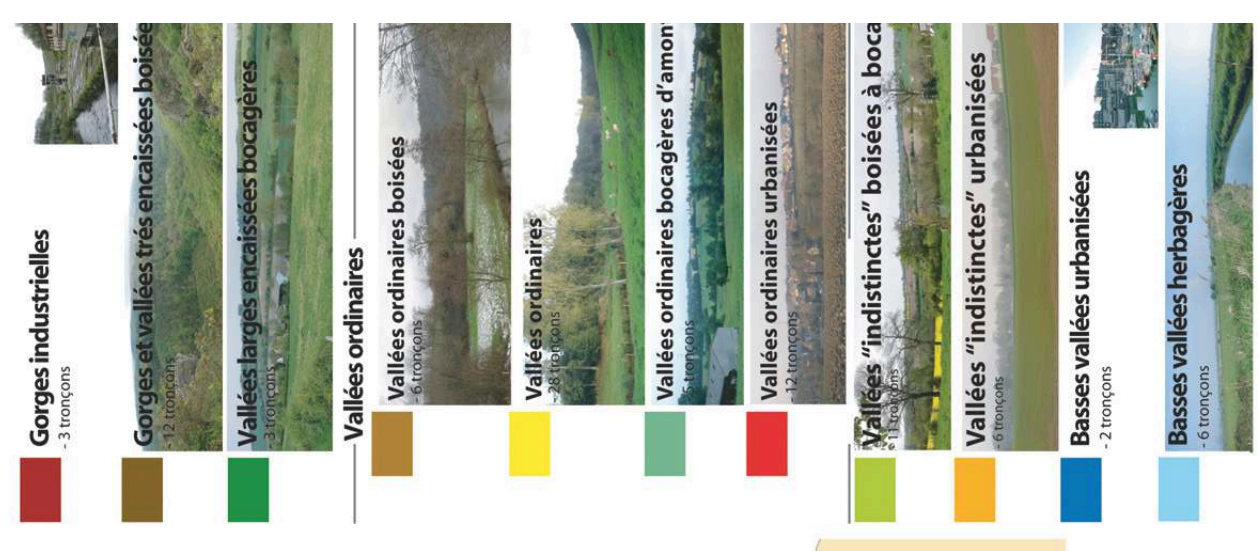

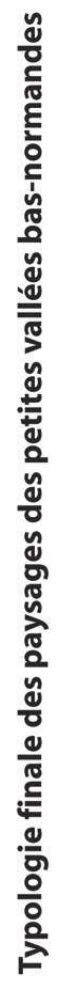

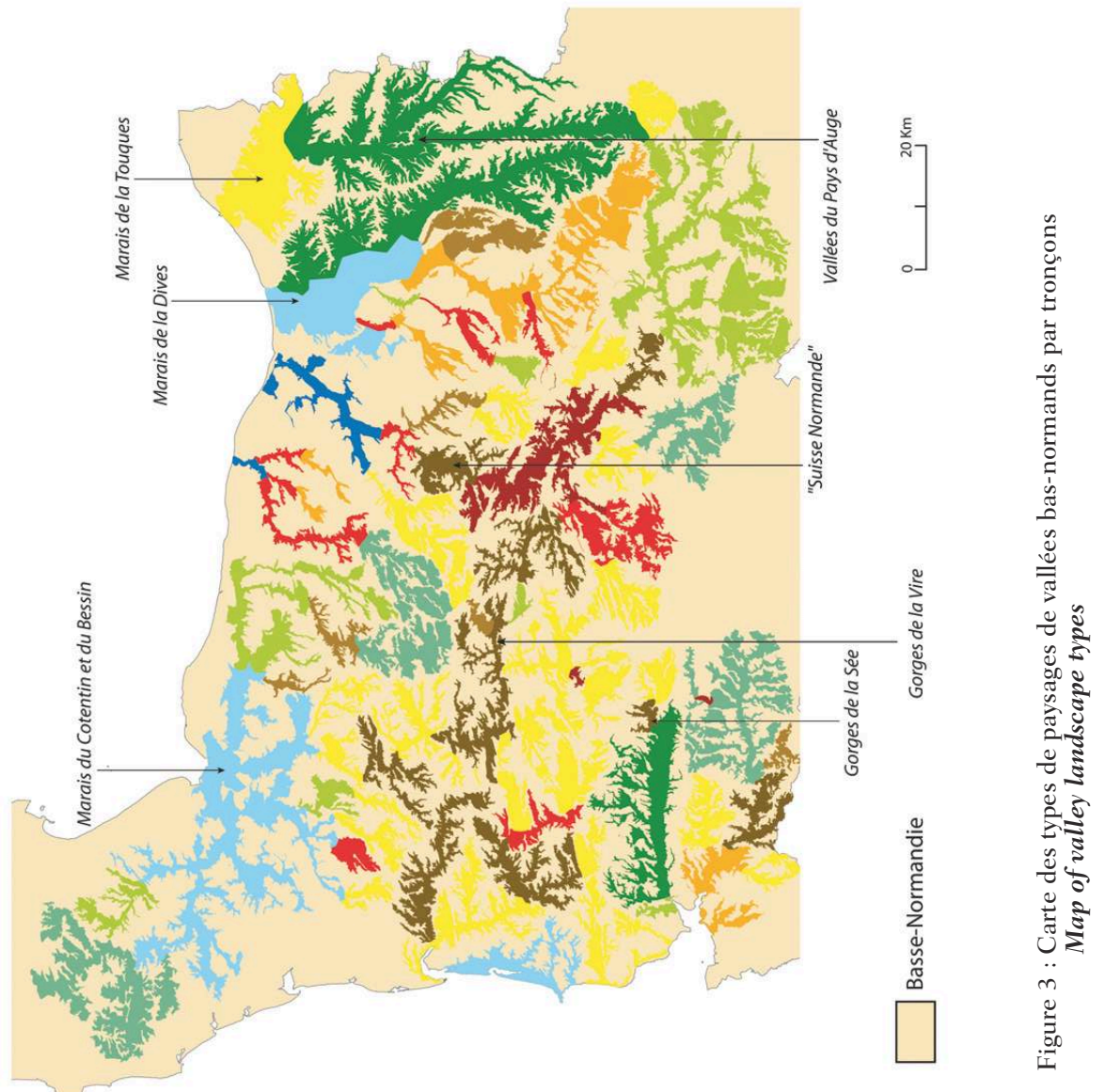

